\title{
Mandibular Condyle Remodeling after Functional Orthopedic Treatment: A Clinical Report of Four Cases
}

\author{
Alain Aube ${ }^{1}$, German O Ramirez-Yanez ${ }^{2}$
}

\begin{abstract}
Aim: This clinical report evaluated changes in the mandibular condyles of patients diagnosed with osteoarthritis (OA) at the temporomandibular joint (TMJ), who were treated with a modified dental splint.

Materials and methods: Four adult patients (age range: 24-47 years) were treated with a dental splint appliance that delivered a low load at the molar region and permitted lateral and protrusive mandibular excursions. An initial magnetic resonance imaging (MRI) scan was performed for diagnosis purpose before treatment. A second MRI scan was conducted after 12 months of treatment to evaluate changes at the TMJ.

Results: The observations from the MRI results in the four patients showed positive changes at the end of the study period. The results presented here suggest the treatment provided significantly reduced the forces damaging the tissues covering the surface of the mandibular condyle, such as cartilage and cortical bone, which may be the cause of the OA. In that context, it is suggested that that reduction in the overloading of the TMJ produced by the functional dental splint permitted the recovery of those tissues.

Conclusion: The present results support the idea that the tissues composing the TMJ are viable and may respond to positive stimulus. In that way, this report proposes a way to treat those patients with TMJ OA, who may respond when the treatment aims to reduce the overloading forces affecting the TMJ.

Clinical significance: This report proposes a noninvasive clinical treatment for patients with TMJ OA, who may respond when the treatment aims to reduce the overloading forces affecting the TMJ.

Keywords: Bone loading, Bone remodeling, Cartilage, Cortical bone, Dental splint, Mandibular condyle, Osteoarthritis, Temporomandibular joint. The Journal of Contemporary Dental Practice (2019): 10.5005/jp-journals-10024-2701
\end{abstract}

\section{INTRODUCTION}

Osteoarthritis is one of the most common forms of arthritis that affects the temporomandibular joint (TMJ). ${ }^{1}$ It affects $10-17 \%$ of patients complaining of pain in the TMJ, although radiological signs of OA may be present in a higher number of individuals., ${ }^{1,2}$ The cause of $O A$ in the TMJ is not fully understood, but its pathophysiology may involve decreased adaptive capacity of the articulating structures or excessive or sustained physical stress on the TMJ that exceeds that normal adaptive capacity. ${ }^{3,4}$

Recently, knowledge regarding how overloading can affect the articular structures of the TMJ leading to OA has improved. ${ }^{5}$ Overloading in the TMJ causes collapse of the synovial lubrication in the joint, which leads to shear stress. Such stress damages the articular cartilage and results in irreversible deformation of the TMJ tissues. $^{6-8}$ Overloading also stimulates the expression of several pro-inflammatory cytokines, such as tumor necrosis factor- $a$, interlukin-1, and interleukin-6, which promote bone resorption by increasing the differentiation and activation of osteoclasts. ${ }^{9}$

Magnetic resonance imaging has been proposed as a standard evaluation to identify degenerative disorders, such as $O A$, in the TMJ. ${ }^{10,11}$ At the mandibular condyle, multiple osseous changes can be identified through MRI. Such changes can include changes in the shape of the condylar head, which may become a flattened, interrupted cortical bone on the surface of the mandibular condyle; deterioration and abrasion of the articular cartilage; and local thickening and remodeling of the underlying bone. ${ }^{12,13}$

Noninvasive management is the most common approach to reduce the symptoms associated with TMJ disorders, including OA. Among these approaches, occlusal splints have been reported to be effective in protecting the TMJ from involuntary overloading and
${ }^{1}$ Private Practice, Institut Canadien d'Occlusion Montreal, Quebec, Canada

${ }^{2}$ Private Practice, Aurora Kids Dentistry, Aurora, Ontario, Canada

Corresponding Author: German O Ramirez-Yanez, Private Practice, Aurora Kids Dentistry, Aurora, Ontario, Canada, Phone: +1 647963 5716, e-mail: ramirezger@gmail.com

How to cite this article: Aube A, Ramirez-Yanez GO. Mandibular Condyle Remodeling after Functional Orthopedic Treatment: A Clinical Report of Four Cases. J Contemp Dent Pract 2019;20(12):1461-1465.

Source of support: Nil

Conflict of interest: None

reducing the pain associated with the disorder. ${ }^{14}$ In this paper, the authors report the use of a flat anterior plane permissive occlusal splint with light posterior contacts to treat the symptoms associated with OA of the TMJ (Fig. 1). The purpose of this clinical report is to present the positive results of four patients diagnosed with OA of the TMJ who were treated with the previously described occlusal splint and evaluated before and after treatment by MRI.

\section{Case Description}

\section{Case 1}

A 35-year-old female patient presented with severe muscular and TMJ pain on the right side of her face. She complained of neck pain and reported daily use of pain medication (methocarbamol and ibuprofen). Her medical history was not relevant, and her dental history included only restorative treatment. She had not undergone orthodontic treatment. 
During the initial clinical examination, she had a limited ability to open her mouth, approximately $23 \mathrm{~mm}$. She had a class I malocclusion associated with an open bite. Muscular palpation resulted in reported pain on the lateral pterygoids on the right side and at the occipital region on both sides.

The initial MRI results (Fig. $2 \mathrm{~A}$ ) revealed anterior and lateral disk displacement without reduction on the right side and medial displacement on the left side, which was associated with mild to moderate $\mathrm{OA}$ of both mandibular condyles. A dental maxillary splint appliance, described below, was designed for her treatment. The appliance had the following features: the primary contact at closing was on the canines with equal loading on both sides. The posterior teeth did not touch the occlusal surface of the splint, resulting in a space between the upper and lower teeth of approximately $20 \mu \mathrm{m}$, which allowed a marking paper to slide through when the patient clenched her teeth on the splint appliance. The incisors contacted the splint appliance, but at a lower load than that on the canines. Lateral mandibular excursions were guided by the canines, and protrusive movements were guided by the incisors. The anterior

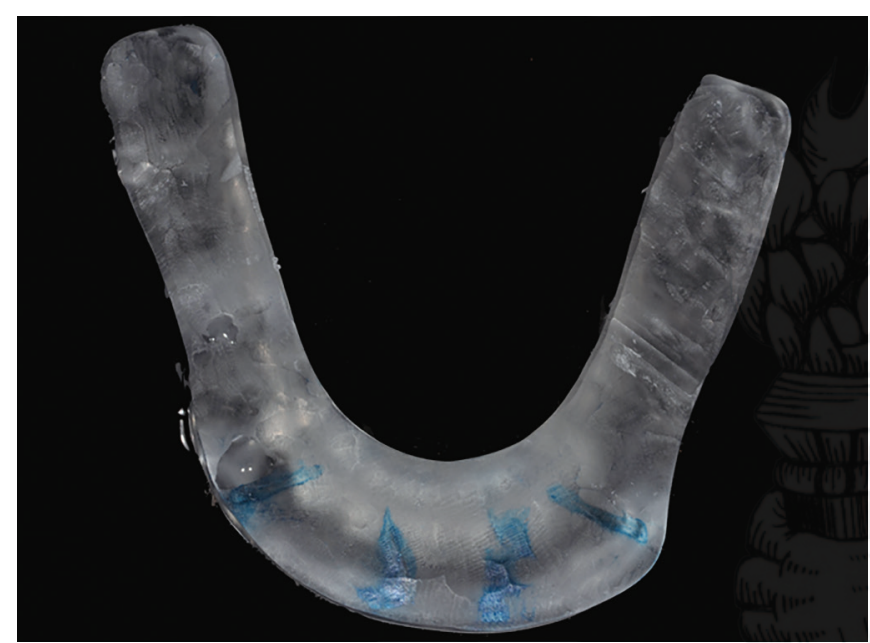

Fig. 1: Photograph of the flat anterior plane permissive occlusal splint used by the authors to treat the signs and symptoms of osteoarthritis diagnosed in the four patients presented here. Note the following features of the appliance: light posterior contacts at the molars and smooth lateral excursions and protrusive movements guided by the canines and central incisors, respectively (blue areas) plane of the splint appliance was flat, and the posterior teeth were not in contact during the mandibular excursions.

The patient was instructed to wear the dental maxillary splint appliance constantly, as long as it did not affect her regular activities. She was also instructed not to use the splint appliance while eating. A follow-up evaluation was performed every 4 weeks to evaluate the symptoms and to adjust the splint to maintain the features described above. The symptoms were evaluated at each monthly appointment via a questionnaire, and a graph showing the presence of the symptoms was plotted (Figs $2 \mathrm{C}$ and D).

An MRI scan was performed 12 months after the start of treatment with the splint appliance, which showed continued anterior disk displacement, but the OA had significantly improved (Fig. 2B). Additionally, the symptoms were reduced after 3 months of treatment, and the patient reported no muscular or TMJ pain at this stage of the treatment. After treating the craniofacial pain symptoms, the patient was referred to an orthodontist to stabilize her dental occlusion, and occlusal equilibration was achieved. The patient has been asymptomatic since treatment with the occlusal splints was completed.

\section{Case 2}

A 47-year-old female patient presented with facial pain on the left side involving the TMJ. Her dental history included the surgical removal of the third molars at 24 years. Her medical history included pharmacological treatment for anxiety.

The dental examination revealed a class II malocclusion with interference during mouth closing at the right molars, which produced a 2-3 mm mandibular shift toward the left side. Muscular palpation resulted in reported bilateral pain in the lateral pterygoid and the temporalis muscles, as well as in the right masseter muscle.

An initial MRI scan showed anterior and lateral disk displacement with partial reduction on the right side but without reduction on the left side. Both TMJs presented moderate OA and capsulitis (Fig. 3A). In addition, the left mandibular condyle presented osteochondritis dissecans with perforation of the cortical bone. After 12 months of treatment with a splint appliance with the same features described above, a second MRI scan was performed. Treatment resulted in a significant improvement of the left mandibular condyle, with no significant changes on the right side. The capsulitis was significantly reduced on both sides, but the disk displacement remained as before (Fig. 3B). At that time, the facial and TMJ pain was not present, and the patient has remained asymptomatic (Figs $3 C$ and $D$ ).
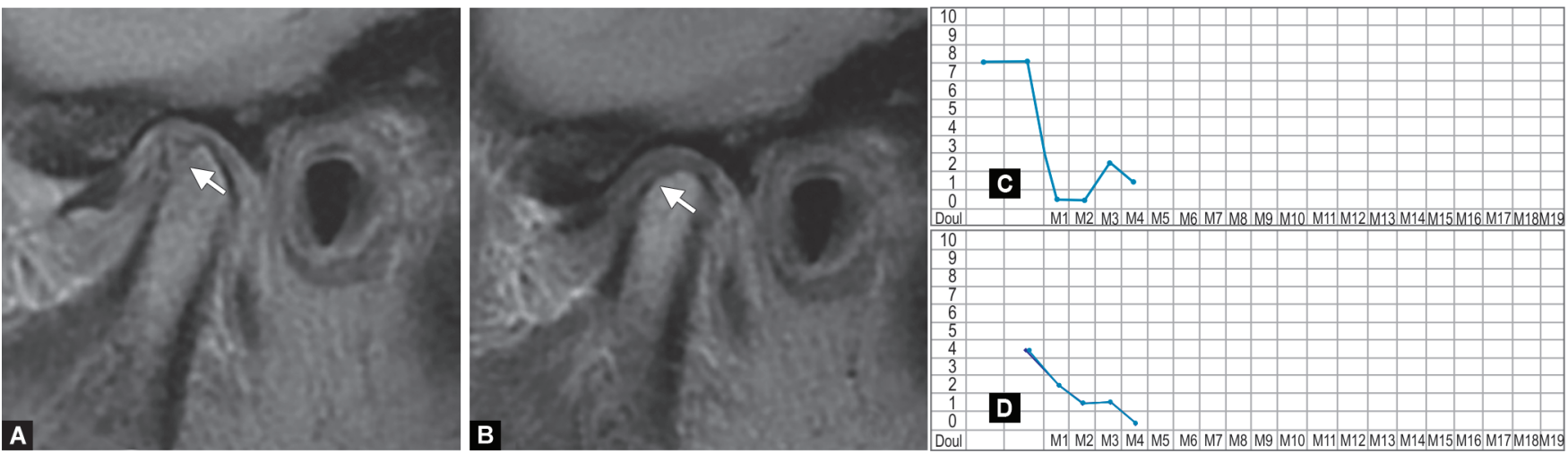

Figs $2 A$ to $D$ : Magnetic resonance imaging results showing the left mandibular condyle before treatment $(A)$ and after treatment $(B)$. Note the irregular surface of the anterior area of the mandibular condyle (A) compared with the smooth and rounded surface of the mandibular condyle after the treatment (B) (white arrows). The graphs recording the monthly changes in the temporomandibular joint (C) and muscular (D) pain in the patient showed a reduction in those symptoms after approximately 4 months of treatment 

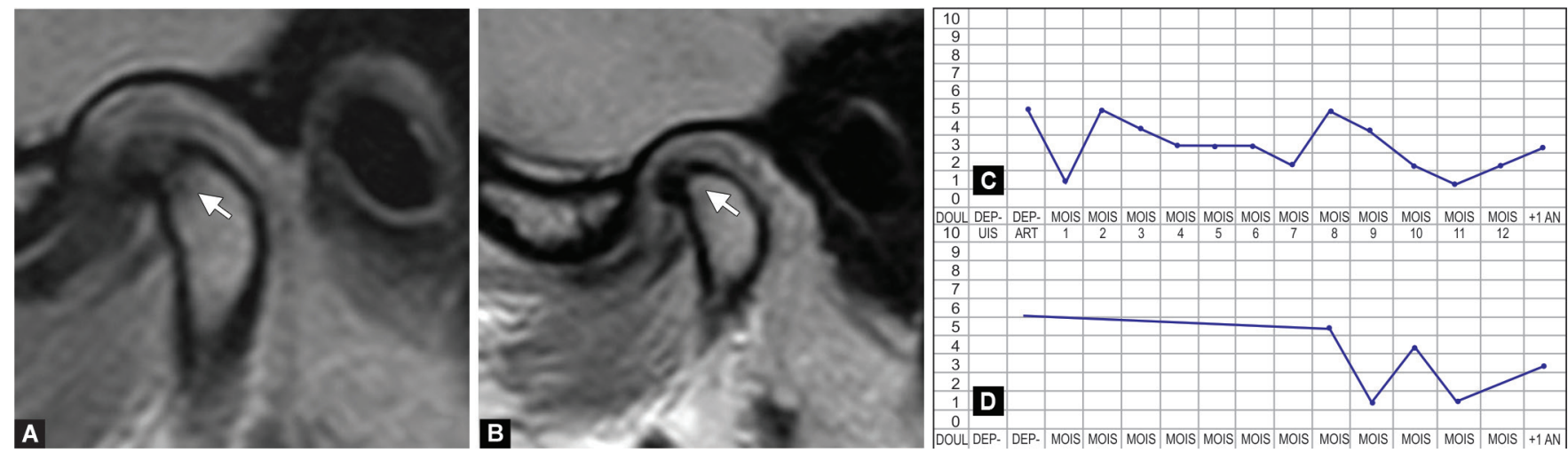

Figs $3 A$ to $D$ : Magnetic resonance imaging results showing the left mandibular condyle before treatment (A) and after treatment (B). Note the bone defect caused by the osteoarthritis associated with osteochondritis dissecans on the anterior area of the mandibular condyle (A) and the smooth surface observed on the mandibular condyle in the same area after the treatment (B) (white arrows). The graphs recording the monthly changes in the temporomandibular joint (C) and muscular (D) pain in the patient showed a reduction in those symptoms at the end of the treatment
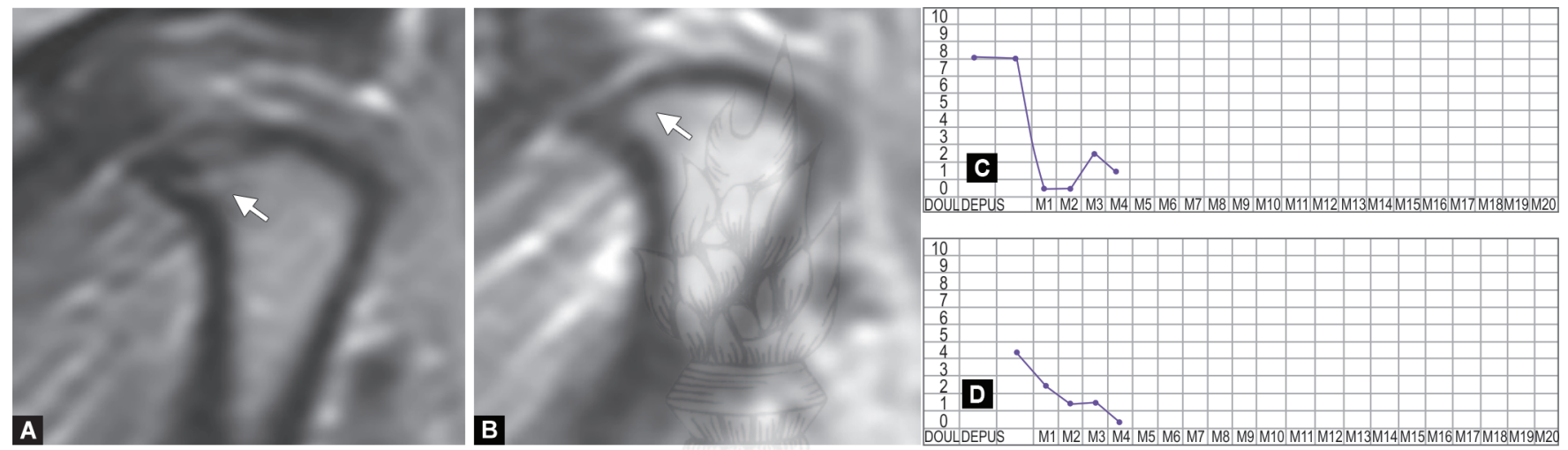

Figs 4A to D: Magnetic resonance imaging results showing the left mandibular condyle before treatment $(A)$ and after treatment (B). Note the irregular and pointy surface caused by osteoarthritis on the anterior area of the mandibular condyle (A), and the smooth and less sharp surface observed on the mandibular condyle in the same area after the treatment (B) (white arrows). The graphs recording the monthly changes in the temporomandibular joint (C) and muscular (D) pain in the patient showed a reduction in those symptoms at 4 months after the initiation of treatment

Along with the improvements reported in the second MRI scan, a significant improvement, compared with the initial MRI results, was observed in the bone perforation caused by osteochondritis dissecans in the left condyle.

\section{Case 3}

A 35-year-old female patient presented with bilateral facial muscular tension and stiffness associated with neck pain on both sides. She reported having a TMJ click on the right side since she was a teenager. Her medical history was not relevant. She also reported that osteopathic maneuvers had improved her condition but did not fix the problem.

A clinical examination revealed a class II malocclusion with dental interference of the right molars during mouth closing. Muscular palpation revealed bilateral pain on the lateral pterygoid muscles on both sides as well as on the right temporalis muscle.

An initial MRI scan revealed anterior and lateral disk displacement without reduction on the right side and anterior disk displacement with reduction on the left side. The results also showed moderate $\mathrm{OA}$ on the right mandibular condyle and osteochondritis dissecans on the same side (Fig. 4A). The patient was instructed to wear the splint appliance with the same instructions as the other patients.
She reported a significant reduction of the symptoms after wearing the splint appliance for 2 months. The treatment continued for 12 months, and a second MRI scan was performed. It showed that the disk displacement continued similarly on both sides, but the OA condition had significantly improved on both sides with significant bone remodeling of the left TMJ (Fig. 4B). Additionally, the osteochondritis dissecans was resolved after treatment.

The patient's dental occlusion was stabilized with orthodontic treatment, and occlusal equilibration was performed afterward. The patient has been asymptomatic since treatment with the referred splints (Figs 4C and D).

\section{Case 4}

A 24-year-old female patient complaining of bilateral muscular tension and fatigue in the temporalis and masseter muscles on both sides presented at the office of one of the authors. Her medical history was not relevant, and she was not taking any medication. She had undergone Lefort I surgery 3 years previously and wore fixed orthodontics for 4 years to correct a class II malocclusion. She noticed that her bite started opening 1 year after the orthodontic/ surgical treatment was completed. At the clinical examination, a class II malocclusion associated with an anterior open bite and 

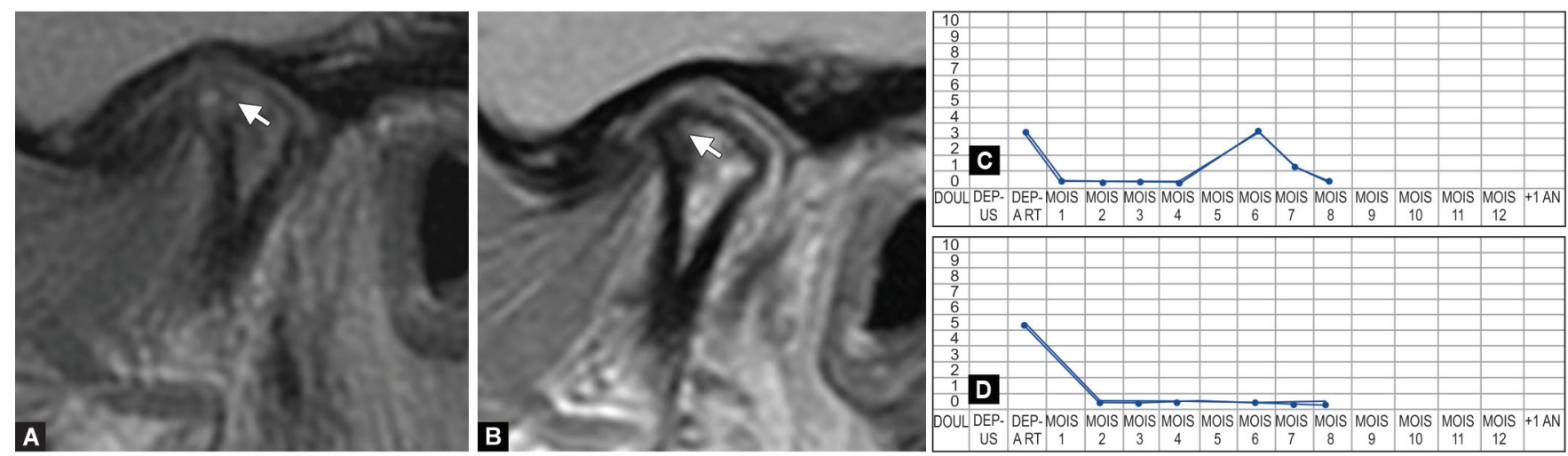

Figs 5A to D: Magnetic resonance imaging results showing the left mandibular condyle before treatment (A) and after treatment (B). Note the irregular surface caused by osteoarthritis on the anterior area of the mandibular condyle (A) compared with the smooth and less broken surface observed on the mandibular condyle in the same area after the treatment (B) (white arrows). The graphs recording the monthly changes in the temporomandibular joint (C) and muscular (D) pain in the patient showed a reduction in those symptoms at 8 months after the initiation of treatment

dental interference on the left molars when closing the mouth was recorded. Muscular palpation resulted in reported pain in the masseter and lateral pterygoid muscles on both sides.

The pretreatment MRI scan revealed anterior disk displacement without reduction in both TMJs along with moderate OA on the right side and severe $\mathrm{OA}$ on the left side (Fig. 5A). A hypoplastic mandibular condyle was observed on both TMJs, with higher severity on the medial portion of the right condyle TMJ (Fig. 5A). The treatment plan involved a dental maxillary splint appliance as described in the first presented case. After 12 months of treatment with the splint appliance, a second MRI scan was performed, which showed that disk displacement without reduction continued on both sides, but a significant improvement in the OA conditions was noticeable with remodeling of the cortical bone on both mandibular condyles (Fig. 5B).

After the initial year of treatment, a partial occlusal equilibration was performed. Currently, the patient is under orthodontic treatment, and she has been asymptomatic since a few months after starting treatment with the splint appliances to the time of publication (Figs 5C and D).

\section{Discussion}

The purpose of this clinical report was to present the improvements in the cortical bone at the TMJ of patients diagnosed with an osteoarthritic mandibular condyle after being treated with a flat anterior plane permissive occlusal splint with light posterior contacts. Those results were observed in MRI studies. The results presented here from four patients suggest that the specific stabilization of the occlusal contacts on the teeth produced by the appliance may produce a reduction in the overloading on the mandibular condyle in the TMJ, which facilitated a remodeling process on the cortical bone at the external surface of the mandibular condyle.

That surface of the mandibular condyle is covered by cartilage that is composed of a fibrocartilage on the more external area and hyaline cartilage immediately underneath. ${ }^{15}$ The mandibular cartilage is responsible for the endochondral ossification generally recognized to participate in the increase in length of the mandible during the growth stages. ${ }^{16}$ The mandibular condyle of adults exhibits an increase in the amount of fibrocartilage while maintaining the hyaline cartilage. ${ }^{17}$ Although morphological changes occur, characteristic cells (fibroblasts) in the fibrocartilage, as well as chondrocytes in the hyaline cartilage, have been observed in the mandibular condyle of adults and elderly individuals. ${ }^{17,18}$

To maintain the integrity of the bone structure of the mandibular condyle, as well as the mandibular dynamics, the endochondral ossification should be maintained. ${ }^{19,20}$ Bone apposition can occur on the joint surfaces only when the articular surface is covered by cartilage. ${ }^{21}$ A recent in vitro study has shown that the fibrocartilage at the surface of the mandibular condyle contains undifferentiated cells, which have the capability to organize in a hematopoietic environment, which may lead to tissue regeneration. ${ }^{22}$ Another report has shown that although the cartilage at the mandibular condyle surface undergoes morphological changes in adults and elderly individuals, it maintains its cellularity in the fibrocartilage and the hyaline cartilage structure. ${ }^{18}$ Those studies support the idea that an osteoarthritic condyle at the TMJ requires a change in the environment by reducing the overloading in situ and allowing the tissue covering the mandibular surface to recover and remodel.

It has been stated in the dental literature that the mandibular condyle losses its growth potential around the pubertal spurt, as the cells in charge of producing the collagen matrix, which may become mineralized, are not present in adults anymore. ${ }^{23,24}$ The clinical results presented here evidence there is still a potential for bone apposition in the mandibular condylar cartilage of human adults. That phenomenon, bone apposition in the mandibular condyle, occurs through endochondral ossification. And, such a process should be leaded by the hyaline cartilage, which contains the chondrocytes, cells able to deposit a collagen matrix which may turn into bone tissue. ${ }^{21}$ A recent study published by one of the authors ${ }^{18}$ reported a layer of hyaline cartilage still present in the mandibular condyle of humans at elderly. In that context, this report supports the idea that the growth potential in the mandibular condyle is present all life in humans and a correct stimulus is necessary for it to express in order to obtain a regenerative response in patients with a degenerative process in the mandibular condyle. Thus, the results presented here suggest that the cartilage tissue covering the surface of the mandibular condyle and containing cells able to respond to different stimuli might be the tissue producing the endochondral ossification that guided the remodeling of the cortical bone observed in the clinical cases presented here. However, further studies are required to better understanding the biology behind the presented clinical results. 
Up today, it is known that overloading at the TMJ during mandibular dynamic motion increases the shear stress at the surface of the mandibular condyle. ${ }^{5,25}$ This negative impact affects the mandibular condyle biology by reducing the synovial lubrication in the joint and physically disrupting the cells in the cartilage, which impairs cellular function, increases the matrix degradation, and inhibits matrix synthesis, resulting in damage and deformation of the TMJ tissues. ${ }^{4,6,8}$ The observations from the four patients presented here suggest that the delivered treatment significantly reduced or even removed the overloading at the TMJ, since the cortical bone initially observed to be damaged in the initial MRI results presented signs of recovery and remodeling in the MRI findings obtained after 12 months of treatment. In that context, we propose that treatment of osteoarthritic condyles associated with symptoms in the TMJ must involve stabilization of the dental occlusion, which reduces overloading in the TMJ. In that context, it is important to remark the appliance should permit light contacts at the molar teeth, as well as smooth lateral excursions guided by the canines and protrusive movements guided by the incisors. Nevertheless, more clinical studies are necessary to demonstrate that the overloading of the TMJ may stimulate condylar degeneration, and conversely, a physiological function may stimulate condylar regeneration.

\section{Conclusion}

This clinical report suggests that the treatment of OA in condyles associated with TMJ disturbances should involve stabilizing the dental occlusion by producing light contacts at the molar region as well as smooth lateral excursions guided by the canines and protrusive movements guided by the incisors. In that way, the overloading of the mandibular condyle may be reduced at the TMJ, and the cortical bone at the mandibular condyle surface may regenerate, reducing the degenerative signs of OA observed through MRI in addition to the clinical symptoms in the patients.

\section{Clinical Significance}

This report propose a way to treat those patients with TMJ OA, who may respond when the treatment aims to reduce the overloading forces affecting the TMJ. It supports the idea that the tissues composing the TMJ may be viable an able to respond in adults, which infers that there is still an opportunity for treating those patients with non-invasive techniques when a degenerative process affects the TMJ.

\section{References}

1. Mejersjö C, Hollender L. TMJ pain and dysfunction: relation between clinical and radiographic findings in the short and long-term. Scand J Dent Res 1984;92(3):241-248. DOI: 10.1111/j.1600-0722.1984. tb00886.x.

2. Zarb G, Carlsson G. Temporomandibular disorders: osteoarthritis. J Orofac Pain 1999;13(4):295-306.

3. Arnett G, Milam S, Gottesman L. Progressive mandibular retrusionidiopathic condylar resorption. Part II. Am J Orthod Dentofacial Orthop 1996;110(2):117-127. DOI: 10.1016/S0889-5406(96)70099-9.

4. Arnett G, Milam S, Gottesman L. Progressive mandibular retrusion: idiopathic condylar resorption. Part I. Am J Orthod Dentofacial Orthop 1996;110(1):8-15. DOI: 10.1016/S0889-5406(96)70081-1.

5. Palla S, Gallo L. Biomechanics and mechanobology of the TMJ. In Greene C, Laskin D, ed. TMDs bridging the gap between advances in research and clinical patient management. Hanover Park, IL: Quintessence Publishing Co Inc.; 2013. pp. 101-112.
6. Tanaka E, Detamore M, Mercuri L. Degenerative disorders of the temporomandibular joint: etiology, diagnosis, and treatment. J Dent Res 2008;87(4):296-307. DOI: 10.1177/154405910808700406.

7. Nickel J, Spilker R, Iwasaki L, et al. Static and dynamic mechanics of the temporomandibular joint: plowing forces, joint load and tissue stress. Orthod Craniofac Res 2009;12(3):159-167. DOI: 10.1111/j.16016343.2009.01449.x.

8. Beatty M, Nickel J, Iwasaki L, et al. Mechanical response of the porcine temporomandibular joint disc to an impact event and repeated tensile loading. J Orofac Pain 2003;17(2):160-166.

9. Boyle WJ, Simonet WS, Lacey DL. Osteoclast differentiation and activation. Nature 2003;423(6937):337-342. DOI: 10.1038/ nature01658.

10. Larheim T. Role of magnetic resonance imaging in the clinical diagnosis of the temporomandibular joint. Cells Tissues Organs 2005;180(1):6-21. DOI: 10.1159/000086194.

11. Summa $S$, Ursini R, Manicone $P$, et al. MRI assessment of temporomandibular disorders: an approach to diagnostic and therapeutic setting. Cranio 2014;32(2):131-138. DOI: 10.1179/08869 $63413 Z .00000000021$.

12. Cortés D, Exss E, Marholz C, et al. Association between disk position and degenerative bone changes of the temporomandibular joints: an imaging study in subjects with TMD. Cranio 2011;29(2):117-126. DOI: 10.1179/crn.2011.020.

13. Oliveira J, Rosa J, Dutra M, et al. Assessing joint effusion and bone changes of the head of the mandible in MR images of symptomatic patients. Braz Oral Res 2013;27(1):37-41. DOI: 10.1590/S180683242013000100007.

14. Kuzmanovic Pficer J, Dodic S, Lazic V, et al. Occlusal stabilization splint for patients with temporomandibular disorders: meta-analysis of short and long term effects. PLoS One 2017;12(2):e0171296. DOI: 10.1371/journal.pone.0171296.

15. Ramirez-Yanez G, Daley T, Symons A, et al. Incisor disocclusion in rats affects mandibular condylar cartilage at the cellular level. Arch Oral Biol 2004;49(5):393-400. DOI: 10.1016/j.archoralbio.2003.11.005.

16. Petrovic A. Control of postnatal growth of secondary cartilages of the mandible by mechanisms regulating occlusion. Cybernetic model. Trans Eur Orthod Soc 1974; 69-75.

17. Luder $\mathrm{H}$. Age changes in the articular tissue of human mandibular condyles from adolescence to old age: a semiquantitative light microscopic study. Anat Rec 1998;251(4):439-447. DOI: 10.1002/ (SICI)1097-0185(199808)251:4<439::AID-AR3>3.0.CO;2-N.

18. Ramirez-Yanez G, Scott J. Architecture of the mandibular Condylar cartilage of elderly individuals: a semiquantitative light microscopic histological study. J Contemp Dent Pract 2019;20:768-772. DOI: 10.5005/jp-journals-10024-2594.

19. Petrovic AG. Mechanisms and regulation of mandibular condylar growth. Acta Morphol Neerl Scand 1972;10(1):25-34.

20. Thilander B, Carlsson G, Ingervall B. Postnatal development of the human temporomandibular joint. I. A histological study. Acta Odontol Scand 1976;34(2):117-126. DOI: 10.3109/00016357609026564.

21. Petrovic A, Stutzmann J, Oudet C. Control processes in postnatal growth of condylar cartilage of the mandible. In: McNamara JAJ, ed., Determinants of mandibular form and growth, Monograph 4th ed., Ann Arbor: University of Michigan; 1975. pp. 14-57.

22. Embree $M$, Chen M, Pylawka S, et al. Exploiting endogenous fibrocartilage stem cells to regenerate cartilage and repair joint injury. Nat Commun 2016;7:13073. DOI: 10.1038/ncomms13073.

23. Katakami K, Shimoda S, Kobayashi K, et al. Histological investigation of osseous changes of mandibular condyles with backscattered electron images. Dentomaxillofac Radiol 2008;37(6):330-339. DOI: 10.1259/dmfr/93169617.

24. Moffett $B$. The morphogenesis of the temporomadibular joint. Am J Orthod 1966;52(6):401-415. DOI: 10.1016/0002-9416(66)90120-5.

25. Tanaka E, Kawai N, Tanaka M, et al. The frictional coefficient of the temporomandibular joint and its dependency on the magnitude and duration of joint loading. J Dent Res 2004;83(5):404-407. DOI: 10.1177/154405910408300510. 